\title{
BMJ Open Quality System approach to prevent lost studies and improve radiology report turnaround time
}

\author{
Jacob Schick, ${ }^{1}$ Jonelle M Petscavage-Thomas (i) ${ }^{2}$
}

To cite: Schick J, PetscavageThomas JM. System approach to prevent lost studies and improve radiology report turnaround time. BMJ Open Quality 2022;11:e001690. doi:10.1136/ bmjoq-2021-001690

Received 5 0ctober 2021 Accepted 13 January 2022
Check for updates

(C) Author(s) (or their employer(s)) 2022. Re-use permitted under CC BY-NC. No commercial re-use. See rights and permissions. Published by BMJ.

${ }^{1}$ Penn State College of Medicine, Hershey, Pennsylvania, USA

${ }^{2}$ Radiology, Penn State Health Milton S Hershey Medical Center, Hershey, Pennsylvania, USA

Correspondence to Dr Jonelle M PetscavageThomas; jopa905@gmail.com

\section{ABSTRACT}

Purpose On our picture archiving and communication system worklist, there was no way to differentiate body imaging (BI) from musculoskeletal (MSK) MR pelvis examinations. They were listed on only the BI worklist. This resulted in 'lost' MSK MR pelvis studies with high report turnaround time (TAT). Some exams had preliminary reports with substantiative changes made days later when found. The goals of this project were to create a solution to prevent 'lost' exams and improve TAT.

Methods A report of 3 months of MR pelvis studies was reviewed to determine time to first view by MSK radiologists, time of completion, time of preliminary report and time of final signature. Mean TAT was calculated and exams with delays in reporting resident misinterpretation recorded.

An MSK reserve flag was created for the BI radiologists to use when they found an MSK study on their worklist. The flag moved them onto the MSK reserve worklist. A second intervention included technologists placing the reserve on examination completion. After this, another 3 months of data was analysed.

Results There was a significant improvement $(p=0.0018)$ in time to view by MSK from preintervention mean of $1125 \mathrm{~min}(\mathrm{n}=107)$ to postintervention mean of $526 \mathrm{~min}$ $(n=127)$. There was also a significant improvement $(p=0.0033)$ in time to view inpatient and Emergency department cases from $927 \mathrm{~min}$ to $357 \mathrm{~min}$. Time from study completion to final signature also improved from a mean of $1764 \mathrm{~min}$ to $838 \mathrm{~min}$, though not statistically significant $(p=0.08)$. There were five cases of delay in reporting resident misinterpretation preintervention and none postintervention.

Conclusion Our intervention shows the importance of modifying human and informatics factors to solve a patient safety issue.Introduction

Final radiology report turnaround time (RTAT) is a commonly used quality metric in academic radiology departments. ${ }^{1}$ It has been ranked by hospital leaders as one of the highest priorities and is defined as timely if it is available to the healthcare team at the time it is needed'. ${ }^{2}$ Delays in image interpretation and RTAT have been shown to increase length of time to appropriate patient management decisions, resulting in higher costs and potential compromises in patient care. ${ }^{3}$ The timely communication of diagnostic studies is also a National Patient Safety Goal of the Joint Commission. ${ }^{4}$

Worklist optimisation is one method of improving workflow efficiency and RTAT within academic radiology departments. ${ }^{56}$ Computerised picture archiving and communication system (PACS) worklists ${ }^{78}$ allow studies to be segmented by modality, subspecialty, location, age, ordering provider and multiple other factors depending on the specific software application. However, there may be examinations that due to current procedural terminology code filtering, are located on multiple worklists, such as an MRI or CT of the pelvis. This type of examination may represent imaging of the prostate, a study for the body imaging (BI) division, or imaging of the hip joint for the musculoskeletal (MSK) division.

In our institution, greater than $80 \%$ of the MR pelvis studies represent BI division studies and the decision was made to exclude these from the MSK worklist as they would clutter that list. Unfortunately, RTAT for MR pelvis studies was found to be an outlier at our institution due to this workflow. There were two sentinel cases reported to our safety team due to 'lost' MSK MR pelvis studies. These cases had a preliminary resident interpretation that was subsequently found to be incorrect, resulting in significant change in patient management. The purpose of this project was to decrease the variability in RTAT for MSK MR Pelvis studies and have zero cases of resident preliminary reads not signed off by 9:00 hours the next day.

\section{METHODS}

Identify and define the process or problem

Two sentinel cases were identified that resulted in delays in patient care due to MR pelvis examination studies on emergency department (ED) patients being in preliminary status on the BI worklist for greater than 48 hours before notification of the MSK radiology division. Audit trails within the PACS 
were reviewed and radiologists involved in the cases interviewed to identify holes in the system process. The defined problem was delay in final reads for MSK MR pelvis studies.

\section{Collect and analyse data to better define the problem}

An initial radiology information system (RIS) report was obtained of RTAT for all MR Pelvis studies performed over a 3-month period. This was subdivided by MR Pelvis studies with final signature performed by an MSK radiologist. Audit logs were reviewed for each study to determine time of examination completion by technologist, time to first view by BI and by MSK radiologists, time of report draft, time of preliminary report and time of final attending signature. Mean times for time from completion to first MSK view, time from completion to preliminary report, time from preliminary report to final signature and time from completion to final report were calculated. Studies with delays in reporting resident misinterpretation were recorded. This was benchmarked to department goals of a mean completion to final signature RTAT of $60 \mathrm{~min}$ for ED and STAT ( $<30$ minutes to be read) inpatient studies.

\section{Identify all possible contributing factors}

A group of stakeholders was identified to assess the problem and create a solution. This consisted of the Vice Chair for Clinical Affairs, a senior resident and an information technology (IT) analyst skilled in workflow optimisation. Division chiefs and senior/chief technologists were also included for workflow questions, feedback and testing of interventions. An initial Ishikawa diagram (figure 1) was created to identify factors contributing to studies remaining on the BI worklist without dictation.

\section{Select the major root causes}

Major root causes identified included both information system and human factors. Information system factors included exams only populating on one worklist, inability to filter exam by order indication or subspecialty at time of

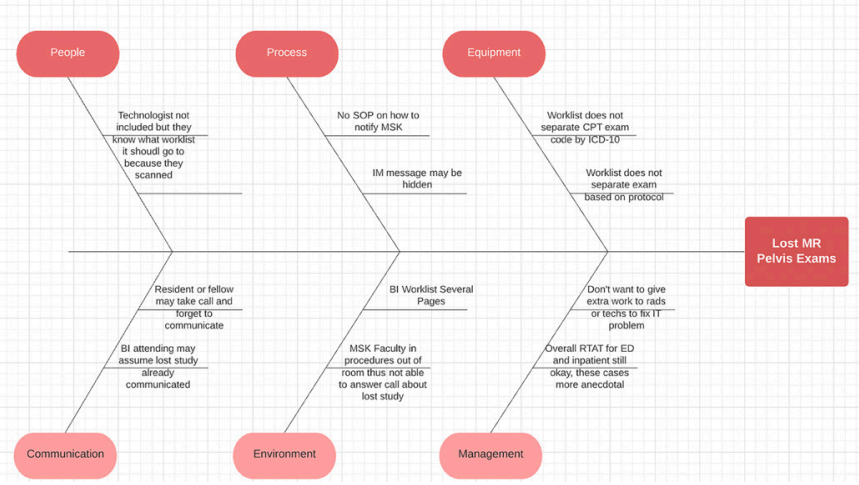

Figure 1 Ishikawa diagram performed to assess factors contributing to the quality problem. CPT, current procedural terminology; ED, emergency department; ICD10, international classification of diseases; IT, information technology; MSK, musculoskeletal; SOP, standard operating procedure.

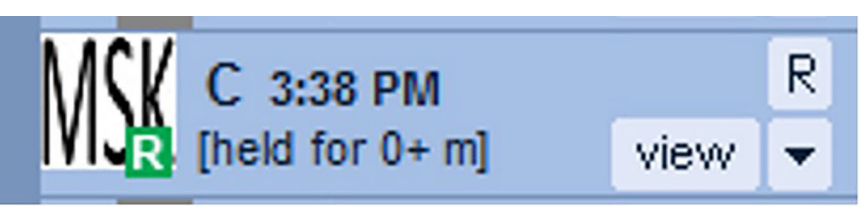

Figure 2 Image shows what the MSK reserve flag looks like on the worklist. MSK, musculoskeletal.

exam completion, and administrative privileges requiring the radiologist to contact IT to get an exam moved to the correct worklist. Human factors included the radiologists assuming someone else would call IT, communication lapses when instant messaging or calling the MSK reading room, inability to find an MSK radiologist in the reading room, and not following department worklist filtering guidelines to include preliminary studies and studies completed within the past 7 days. Some radiologists only allowed studies performed within the past 24 hours to populate their worklists, thus, these 'lost studies' dropped off of their lists. Others did not include resident preliminary reads on their filters, thus missing these cases.

\section{Develop solution strategies}

First intervention was implementation of an 'MSK' reserve flag in the worklist. Any resident, fellow or attending can right click on the exam in the worklist and add the reserve flag (figure 2). The BI division was asked to make this intervention when an MSK MR Pelvis was identified on their list. The flag left the study on the BI worklist but identified to the BI radiologists that it was not to be read by them. This decreased the inefficiency of opening the study by multiple BI attendings/residents.

The flag duplicated the study onto a newly created MSK Reserve worklist. The MSK radiologists were asked to read off of their normal MSK CT/MR/US and plain film lists as well as the new MSK reserve list. In the worklist application (Nuance Primordial Prism), these merge into one larger list, thus the radiologist does not need to flip between lists.

The BI and MSK division were also asked to expand their filters to greater time periods and in the morning include preliminary reads so not to miss the overnight ED resident reports.

\section{Implement, test and evaluate the solutions}

After 3 months, another RIS report was collected and analysed. Studies were still 'lost'. Contributing factors included the MSK rads not selecting the MSK reserve filter into their favourites list and MSK residents opening the studies and either dictating or closing due to complexity, thus allowing the reserve flag to disappear. The application could not be coded to keep the flag on once 'claimed' by a user.

Two additional solutions were then implemented. This included having the MR technologist select the reserve flag at time of examination completion. The technologists could perform this by right clicking on the image in the PACS and selecting reserve, MSK. This would ensure 
at time of completion, study landed on the correct list. The radiology residents were also instructed to select the reserve tag after preliminary read to push the study onto the correct worklist. Finally, the BI attendings were instructed that the new standard operating procedure was to also call or instant message the MSK attending if they found an ED or inpatient STAT examination that they were flagging.

\section{Reanalyse processes and repeat steps}

After 3 months, another RIS report was run and analysed in the same methods as prior reports to determine current TAT and identify any 'lost' studies.

\section{RESULTS}

Preintervention time to view any MR Pelvis by MSK attending was $1125 \mathrm{~min}(\mathrm{n}=95)$. Time to view inpatient and ED cases was $927 \mathrm{~min}$. Five cases of delay in reporting resident misinterpretation were found preintervention with mean time of preliminary report to final signature of 1064 min (range 5-7182).

At final implementation, there was a significant improvement $(\mathrm{p}=0.00108)$ in time to view by MSK to postintervention mean of $526 \mathrm{~min}(\mathrm{n}=119)$. There was also a significant improvement $(\mathrm{p}=0.0033)$ in time to view inpatient and ED cases to $357 \mathrm{~min}$. Time from study completion to final signature also improved from a mean of $1764 \mathrm{~min}$ preintervention to $838 \mathrm{~min}$ postintervention, though not statistically significant $(\mathrm{p}=0.08)$. There were zero cases of delay in reporting resident misinterpretation postintervention. The mean time to view overnight preliminary reports improved by $198 \mathrm{~min}$ after intervention. Figure 3 summarises results.

Further analysis was performed stratifying time to view by MSK attending by the clinical site where the MR study was performed. Altogether, our institution receives MR pelvis studies from four clinical sites including the main academic hub. Although the mean time to MSK read was decreased for studies performed at each clinical site, only MR studies performed at the main hub were found have a significant reduction in time to MSK attending view

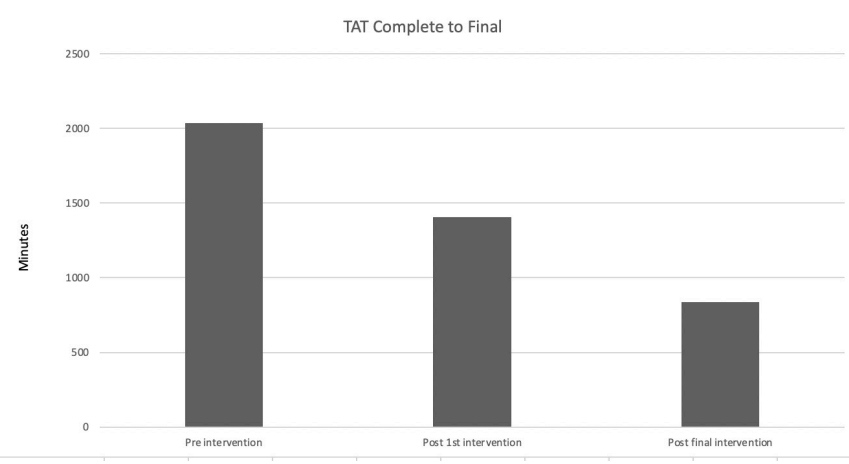

Figure 3 Bar chart shows mean RTAT prior to each intervention and final RTAT after all interventions. TAT, turnaround time; RTAT, Report turn around time. $(\mathrm{p}=0.006)$. This is the site of original sentinel cases and only site with ED and inpatient classes.

In the subsequent months, a 3-month RIS report showed that report turn around time (RTAT) slightly worsened due to presence of new faculty and fellows who were new to the workflow. After training, RTAT improved for MR Pelvis studies. The same model was applied to MR Pelvis studies that were of the lumbosacral plexus. A Neuro reserve tag was created to address this potential 'lost' study.

\section{DISCUSSION}

Multiple prior studies have shown improvement in RTAT by implementation of PACS and speech recognition software. ${ }^{9-11}$ RTAT has also been improved through workflow modification and individual accountability. ${ }^{2}{ }^{12}$ Our study similarly demonstrates that both technology and human factors play critical roles in process improvement of RTAT. In our initial fishbone diagram, multiple system and human factors were identified with realisation that there was not a single solution.

In the BI reading room, lists are often several pages due to the volume in our multi-hospital system. Additionally, there are both in hospital and remote readers and proceduralists, thus communication barriers exist in discussing who will call IT, who will call MSK, and who is available in MSK to chat with. Some BI imagers preferred to send an instant message in PACS rather than communicate by telephone. It was found that these instant messages sometimes hide behind other windows and are never seen. However, the BI attending believed their communication job was done. Additionally, a resident who took a call about a lost study may take the message, but go off to a procedure or conference, and that message about the case gets delayed in communication to the MSK radiologist.

In the final implementation, the $\mathrm{BI}$ attendings were only asked to communicate about ED or inpatient studies, in order to lessen the burden of potential calls. They were also asked to communicate attending to attending and ensure a repeat back/confirmation that communication was received. The hope was this would decrease the human factor in the equation.

Worklist management can be robust, allowing filtering of examinations by modality, subspecialty, examination code, ordering provider, study indication, patient age, patient location and multiple other factors. Unfortunately, as the detail increases, so does the risk of missing a study and errors resulting, creating 'lost' cases. In our institution, it was determined that filtering deeper to try to send MSK MR pelvis studies would miss cases or incorrectly assign some to the MSK list when they were BI or Neuroradiology indications. Thus, further filtering was not a better solution.

The reserve flag was an innovative means of forcing the studies to populate another worklist while having a visual cue on the BI list that they were not to be touched 
by BI faculty. The ease of the reserve flag was a two click mechanism, making adoption by the end user successful. Additionally, the ability of any end user to perform the task made adoption acceptable, especially compared with prior need to call the information services team to move the study to the correct list. It is important to provide a yearly reminder to the faculty to refresh understanding of the policy. It is also imperative to educate new faculty that have joined since policy implementation.

Adding the layer of technologist flagging allowed outpatient studies and those that don't often receive a resident prelim report to readily populate to the MSK list. Adding the resident layer of flagging after issuing a preliminary report also provided assurance that the prelim reads did not fall back onto the wrong worklist. These were the cases identified in the initial discovery period as the highest risk because many radiologists see the preliminary read and if not assigned to cover the overnight cases, only filter their worklists to see completed studies. This decreased visibility initially led to studies sitting for up to a week in the preliminary status.

As the outcomes show, plan-do-study-act (PDSA) cycles and continued reassessment of targeted outcomes is important to ensure other system factors are not contributing and that interventions are working as planned. The success of this intervention allowed adoption of the reserve flag solution to neuro, paediatric and ultrasound cases within our worklists. Another important factor to success was buy-in from stakeholders. Including a technologist in the discussion enabled agreement that the flagging was feasible for the technologists. Including a resident verified their overnight workflow and potential barriers to success. Finally, educating new users to the procedures is important in maintaining targets and providing optimal patient care.

Limitations of our study include retrospective nature of TAT at 3-month intervals rather than more frequently. Limitations also include no monitoring of the communications between BI and MSK, thus not validating if that part of the improvement project was as successful as the informatics solution. Future steps may include use of machine learning to identify cases at risk of extended RTAT and use of AI for drive studies to correct worklists. ${ }^{13}$

\section{CONCLUSION}

Our quality project used IT tools and modified human factors to improve RTAT for MSK MR Pelvis examinations and to result in zero cases of delayed communication of resident misinterpretation of overnight examinations. The reserve flag is a unique function that could be replicated at other institutions looking to help with worklist management.

Contributors We have both contributed to the manuscript and approved its contents. JT is the guarantor.

Funding The authors have not declared a specific grant for this research from any funding agency in the public, commercial or not-for-profit sectors.

Competing interests None declared.

Patient and public involvement Patients and/or the public were not involved in the design, or conduct, or reporting, or dissemination plans of this research.

Patient consent for publication Not required.

Provenance and peer review Not commissioned; externally peer reviewed.

Data availability statement All data relevant to the study are included in the article.

Open access This is an open access article distributed in accordance with the Creative Commons Attribution Non Commercial (CC BY-NC 4.0) license, which permits others to distribute, remix, adapt, build upon this work non-commercially, and license their derivative works on different terms, provided the original work is properly cited, appropriate credit is given, any changes made indicated, and the use is non-commercial. See: http://creativecommons.org/licenses/by-nc/4.0/.

ORCID iD

Jonelle M Petscavage-Thomas http://orcid.org/0000-0001-6517-9732

\section{REFERENCES}

1 Walker EA, Petscavage-Thomas JM, Fotos JS, et al. Quality metrics currently used in academic radiology departments: results of the QUALMET survey. Br J Radiol 2017;90:20160827.

2 Towbin AJ, lyer SB, Brown J, et al. Practice policy and quality initiatives: decreasing variability in turnaround time for radiographic studies from the emergency department. Radiographics 2013;33:361-71.

3 Berlin L. Communicating radiology results. Lancet 2006;367:373-5.

4 Ref2: joint Commission. National patient safety goals, 2021. Available: https://www.jointcommission.org/-/media/tjc/documents/ standards/national-patient-safety-goals/2021/npsg_chapter_hap_ jan2021.pdf

5 Erickson BJ, Meenan C, Langer S. Standards for business analytics and Departmental workflow. J Digit Imaging 2013;26:53-7.

6 Meenan C, Erickson B, Knight N, et al. Workflow Lexicons in healthcare: validation of the swim lexicon. J Digit Imaging 2017;30:255-66.

7 Reiner B, Siegel E, Carrino JA. Workflow optimization: current trends and future directions. J Digit Imaging 2002;15:141-52.

8 Reiner BI, Siegel EL, Not Available NA. The cutting edge: strategies to enhance radiologist workflow in a Filmless/Paperless imaging department. J Digit Imaging 2002;15:178-90.

9 Twair AA, Torreggiani WC, Mahmud SM, et al. Significant savings in radiologic report turnaround time after implementation of a complete picture archiving and communication system (PACS). J Digit Imaging 2000;13:175-7.

10 Mehta A, Dreyer K, Boland G, et al. Do picture archiving and communication systems improve report turnaround times? J Digit Imaging 2000;13:105-7.

11 Lepanto L. Impact of electronic signature on radiology report turnaround time. J Digit Imaging 2003;16:306-9.

12 Deitte LA, Moser PP, Geller BS, et al. Email notification combined with off site signing substantially reduces resident approval to faculty verification time. Acad Radiol 2011;18:774-81.

13 Shah V, Chillakuru YR, Rybkin A, et al. Algorithmic prediction of delayed radiology Turn-Around-Time during Non-Business hours. Acad Radiol 2021;S1076-6332:00265-8. 